\title{
A Study on the Application of Chinese Motivation in International Chinese Education
}

\author{
Nan-nan Wang \\ School of International Exchange, Anshan Normal University \\ Liaoning, China \\ wangnannan@hanmail.net
}

\begin{abstract}
With the emergence of cognitive linguistics, motivations between the form of linguistic symbols and their demonstrative meanings have been found by linguists. Although great achievements have been made in the study of motivation in Chinese, it is mainly focused on the analysis of linguistic phenomena and lacks of application and practice. Aiming at this problem, this paper, combining the research of Chinese motivation with international Chinese education, discusses the application and effect of Chinese motivation in international Chinese education and provides a new method and reference for international Chinese education and teaching.
\end{abstract}

Keywords-Arbitrariness; Motivation; Teaching Chinese as a foreign language; Application research

\section{INTRODUCTION}

Since Saussure argues that the relationship between signifier and signified of linguistic symbols is arbitrary, and the arbitrariness of language has always been regarded as the dominant principle of linguistic symbols. However, with the development of language research, it can be found that some language symbols have some motivations, especially with the rise of cognitive linguistics, the motivation of language has become an important subject in language research. Cognitive linguists believe that the structure and meaning of language are generally justified rather than arbitrary without any inevitable connection. Although Chinese teachers as a foreign language have more or less shown the motivation of Chinese to the learners in actual teaching, most of them are to increase the interest of learning Chinese, the randomness is relatively large, and lack of theoretical basis. Based on the relevant theories of language motivation, this paper combines the Chinese motivation with the teaching Chinese to speakers of other languages, in order to achieve the purpose of improving the teaching effect of teaching Chinese to speakers of other languages.

\section{THE CONCEPT OF MOTIVATION AND ITS FUNCTIONAL FACTORS}

Many scholars have interpreted the concept of language motivation, some of which have been defined as follows:

(1) Haiman (1980/1985) [1-2]: motivation refers to the structural similarity between language and conceptual entities.

(2)Lakoff (1987) [3]: motivation refers to the demonstrable association $\mathrm{L}$ between $\mathrm{A}$ and $\mathrm{B}$.

(3)Hiraga (2005) [4]: motivation refers to the non-arbitrary relationship between form and meaning.

It can be seen from (1) that although linguists have different definitions of motivation, the relationship between the form and content of language is not arbitrary, but related to each other. That is, if the relationship between the form and the content of a language can be explained, we can say that it is motivated.

Based on the above definition and the study of Radden \& Panthera (2004) [5], we conclude the main factors of language motivation as three: The first is empirical motivation. In cognitive linguistics, the image schemas formed by body experience is a form of motivation given to linguistic units. For example, the concepts of "up" and "down" are formed by our personal experience under the influence of earth gravity, and the concept of quantity in many languages can be expressed through "up" and "down". The reason is that the increase of the number of objects in daily life is accompanied by the increase of the height, and the decrease of the number of objects is basically accompanied by the decline of the height of the body experience. The second is cognitive motivation, different cognitive styles, will make language show different forms of expression. Just as an object "xiang-zao" in Chinese is called "toilet soap" in English, "ke-sho-u-se-kke-n" in Japanese, "sesus-pi-nu" in Korean, reflects the cognitive subject's cognition of "soap" of the same object, different prominence is chosen. Chinese has highlighted its taste, English has highlighted the object of its use, Japanese and Korean have highlighted their different functions. The third is the ecological machine, all things have their own biological characteristics. Such as "huaban(petals)", "hua-cha(scented tea)", "hua-wen(pattern)" of Chinese indicated by the form of "flower+X" form of naming 
depends on the object itself concept structure, where " $\mathrm{X}$ " is respectively "flower" composition, "flower" function, "flower" shape. It can be seen that language forms and what they indicate are not unscrupulously in most cases, but have an inseparable relationship with our experience, cognition, and the characteristics of the indicative object itself.

\section{The Chinese Motivation AND THE Teaching OF CHINESE TO SPEAKERS OF OTHER LANGUAGES}

According to the summary, the arbitrariness of language is shown only when language units are regarded as independent individuals, and the motivation of language is the characteristic that occurs when we examine the arrangement and combination of language units. When we teach Chinese to learners, showing the Chinese motivation is not only beneficial to students' understanding and memory, enlarging their vocabulary, consolidating and deepening their grammar knowledge, but also improving their ability to discriminate and understand Chinese. It even enables learners to discover some Chinese rules autonomously. This article will mainly discuss how to apply the Chinese motivation in the teaching Chinese to speakers of other languages from the following three aspects in order to improve the teaching effect of Chinese as a foreign language.

\section{A. The application of the meaning of words' motivation in vocabulary teaching}

The traditional teaching of Chinese as a foreign language attaches great importance to grammar teaching, but neglects the improvement of students' vocabulary and the training of language communicative competence. However, vocabulary is not only the carrier of grammar, but also the loaded information of culture and language, the sufficient vocabulary, the understanding of the meaning of words and the mastery of the usage of words, that directly affect the students' communicative competence, therefore, vocabulary teaching is an important factor in the success of foreign language learning. At present, vocabulary teaching mainly adopts direct explanation by teachers, teachers often use teaching methods such as visual demonstration, contrastive analysis, morpheme interpretation and so on. However, these methods emphasize the literal meaning of words too much, ignore the extended meaning and words' motivation, the result is that learners do not know how to use the extended meaning of the words, and memorize them blindly. From the perspective of cognitive linguistics, the extended meaning of words is evolved by different paths from the basic meaning of words, that is, there is motivated between the basic meaning and the extended meaning. For example, the basic meaning of the noun "tou (head)" is "the uppermost part of the human body or the front part of the animal body". "tou(head)" in "ti-tou(have one's head shaved)" means "hair", "tou(head)" in "shantou(mountain head)" means "top", "head" in "cong-tou(from the beginning)" means "starting point" and so on; The basic meaning of the verb "chi(eat)" is "to swallow things in the mouth", but in this towel is "chi-shui(draught)", there is the meaning of "absorption" and in "chi-diao(destroy)" a company of the enemy, there is the meaning of "annihilate" and so on. There is a certain proximity or similarity between the basic meaning and the extended meaning, such as "hair" is a part of "head", in the special context of "shan-tou (mountain head)", the meaning of "hair" is activated; the basic meaning and the extended meaning of "eat" act on two objects, both of them have the characteristics that one object enters another. If the teacher can show the learner the process in which they develop the extended meaning from the basic meaning, that is, they are the motivation to establish a certain relationship between the similarities between two things in the human brain and then reflect the result through the vocabulary. It not only makes words themselves fresh and interesting to students, but also greatly improves the efficiency of learners' memorization of vocabulary. [6] Cognitive semantics regards "metonymy" and "metaphor" as the main mechanism of word meaning expansion. Mastering the principle of motivation of these words will also help to improve learners' ability to distinguish and analyze the meaning of words. For example, for the word "xi-qian (money laundering)", if students can extract the information that "xi (wash)" has "remove dirt through water", and add the text before and after, it would not be difficult to infer that the term "xi-qian" has the semantic connotation of "legalizing illegally acquired money through some means". In addition, the semantics of many Chinese compound words can not be directly inferred from the morphemes that make them up, teachers should pay more attention to the explanation of these etymological motivations in normal teaching so as to deepen the learners' memory. For example, the word "pai-mapi (flattery)" originated from a culture of the Yuan Dynasty, that is, when ordinary people of the Mongolian nationality met each other with horses, they often patted each others' horses' buttocks in order to win the favour of the horse owners. Later people gradually used this word to describe flattery or applepolish to others. Although the study of Chinese vocabulary is of great significance to the teaching of Chinese vocabulary as a foreign language, we should have a scientific attitude in teaching the motivations of the words, bearing in mind subjective assumptions and paying attention to grasping the yardstick at the same time, there is no need for all words to be presented on a motivational basis.

\section{B. The application of word order motivation in grammar teaching}

Chinese word order is the most important means of grammatical expression in Chinese. In previous teaching, teachers often only pay attention to the explanation of grammatical knowledge and neglect the cultivation of learners' transformation of these abstract grammar rules into language use. When teaching grammar rules, if teachers can make students feel these seemingly complicated grammar rules from their own experience, students will not find Chinese grammar boring. On the contrary, it is an effective way to accept, understand and apply these abstract grammatical knowledge in a more relaxed environment, and to teach grammar according to the motivation of Chinese word order. For example, when we talk about serial-verb construction, combine the structure of "shang-ke(attend class)" and "qu-xuexiao(going to school)" into a sentence, we can remind learners to think about which one is before and which is after of "attend class" and "going to school", learners can organize sentences according to the actual sequence of events. Teaching Chinese word order with the aid 
of the temporal iconicity principle of the existence of Chinese word order in this way can not only deepen students' understanding of Chinese word order, at the same time, but also answer the question of why English can speak and Chinese can only express them in the opposite order [7]. For example, the sentences "laoshi-zhan-zai-le-jiangtai-Shang" and "laoshizai-jiangtai-shang-zhan-zhe" can also be used to explain the inherent word order problem by the principle of temporal iconicity. The former "jiangtai (platform)" is the end point of the teacher's action of "zhan (stand)". In the latter, the teacher exists on the platform in the state of "zhan (stand)". The former observes the relevant scenes in the order of "laoshi (teacher)" to "jiangtai (platform)", and describes the execution process of an action. The latter observed the relevant scenes in the order of "platform" to "teacher" and described the continuous process of a certain state. It can be seen that the principle of temporal order can fully explain the semantic differences lead to the difference of word order in expression. Explaining the rules of Chinese grammar from the motivation of word order can not only better reveal the rules of Chinese grammar, but also arouse the curiosity of learners to explore Chinese grammar, so as to produce the interest of comparing the similarities and differences between Chinese and mother language.

\section{The application of motivation in Chinese culture teaching}

The main teaching content of cultural teaching includes idiom allusions, aphorisms, new words, idioms, metaphors, acronyms, abbreviations and so on. Through the introduction of these knowledge, learners can understand the unique aesthetic concept of Chinese culture, Chinese thinking habits and values, modern and contemporary China's national conditions and so on. However, the teaching of many cultural factors can run through the teaching of pronunciation, Chinese characters, vocabulary, to achieve the goal of teaching in fun. For example we can properly explain the homophonic phenomenon in Chinese in phonetic teaching, and then introduce the relevant cultural background, because many traditional homophonic phenomena are rooted in national culture. This will enable learners to learn and master some cultural knowledge while learning about the language. Such as the ancient name taboo can be divided into national taboo, constitutional taboo and family taboo, in the use of it is necessary to avoid, if there is a related homonym in the speech is to be punished. For example, a scholar in Qing dynasty lost his life because of the homonym of "qing" of "qing-feng (cool breeze)" and "qing" of "Qing dynasty" in poems. Another example is that "hua-sheng (peanut)" is the most common blessing carrier in Chinese traditional weddings. This homonym has the interaction between language and culture, that is, using the homonym of "hua-sheng" as a concrete thing has a deep meaning, there is a traditional Chinese concept of many children and many blessings. In addition, Chinese characters originated from hieroglyphics and are motivated. In Li Baogui(2005) elaborated the performance of the motivation of Chinese characters from the angles of sound side and shape side respectively, which showed that the modern Chinese characters still maintained a high motivation, and talked about the motivation of simplified characters. This is also a problem that we can not avoid when applying the motivation of Chinese characters to the teaching of Chinese characters in foreign countries.[8] In the teaching of Chinese characters, showing the motivation of Chinese characters can also properly interspersed with some cultural content in order to improve learners' memory and interest in Chinese characters. For example, by showing the composition and origin of the Chinese character "fu(father)", it is not only convenient for learners to remember, but also can convey that "father" contains the cultural meaning of manual labor by means of tools in ancient times. In addition, there are many cultural words in Chinese. When explaining these cultural words, it is best to attach them to a certain cultural context of Chinese, which is related to the cultural background of Chinese, so as to increase the interest of learners, at the same time improve their acceptance of Chinese and their ability to communicate in Chinese.

\section{CONCLUSION}

From the angle of teaching Chinese to speakers of other languages and teaching, this paper tries to integrate the Chinese motivation into the teaching of Chinese international education on the basis of previous researches on motivation theory, and discuss the application of Chinese motivation mainly from the vocabulary teaching, grammar teaching, culture teaching three aspects, so as to improve the application of Chinese motivation in the teaching of Chinese international education. However, the demonstration of motivation in this paper is still very onesided, and its practice in teaching Chinese as a foreign language remains at the surface. How to better integrate the motivation into the teaching of Chinese international education remains to be explored and deeply studied by more teachers and researchers of Chinese as a foreign language.

\section{REFERENCES}

[1] Haiman,J. The Iconicity of Grammar. Language, 1980(56)

[2] Haiman,J. Iconicity in Syntax. John Benjamins Pub, 1985.

[3] Lakoff, G. Women, Fire and Dangerous Things: What Categories Reveal About the Mind, Chicago: The University of Chicago Press, 1987.

[4] Radden, G. \& K-U. Panther. Introduction: Reflections on motivation. in Radden \& Panther(eds.), Studies in Linguistic Motivation, Berlin: Mouton de Gruyter, 2004.

[5] Hiraga, Masako.k. Metaphor and Iconicity: A Cognitive Approach to Analysing Text. Palgrave Macmillan, 2005.

[6] Tian Yongfang. The Motivation of Word and its Applied in Teaching Chinese as a Foreign Language. Journal of Guangxi Normal University for Nationalities, 2013(6).

[7] Li Wei. Cognitive study of Modern Chinese word order and Teaching Chinese as a Foreign language. Master's degree thesis of Heilongjiang University, 2011.

[8] Liu Lin. The Motivation of Chinese Characher and Studying of Teaching Chinese Character to Foreigners. Master's degree thesis of Liaoning Normal University, 2011. 